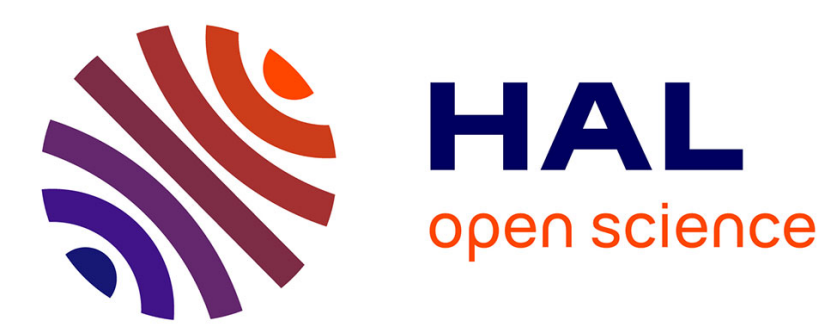

\title{
Dimensional transition of energy cascades in stably stratified forced thin fluid layers
}

A Sozza, G Boffetta, P Muratore-Ginanneschi, Stefano Musacchio

\section{To cite this version:}

A Sozza, G Boffetta, P Muratore-Ginanneschi, Stefano Musacchio. Dimensional transition of energy cascades in stably stratified forced thin fluid layers. Physics of Fluids, 2015, pp.035112. 10.1063/1.4915074 . hal-01144174

\section{HAL Id: hal-01144174 \\ https://hal.univ-cotedazur.fr/hal-01144174}

Submitted on 21 Apr 2015

HAL is a multi-disciplinary open access archive for the deposit and dissemination of scientific research documents, whether they are published or not. The documents may come from teaching and research institutions in France or abroad, or from public or private research centers.
L'archive ouverte pluridisciplinaire HAL, est destinée au dépôt et à la diffusion de documents scientifiques de niveau recherche, publiés ou non, émanant des établissements d'enseignement et de recherche français ou étrangers, des laboratoires publics ou privés. 


\section{AIP $\mid$ Physics of Fluids}

\section{Dimensional transition of energy cascades in stably stratified forced thin fluid layers}
A. Sozza, G. Boffetta, P. Muratore-Ginanneschi, and S. Musacchio

Citation: Physics of Fluids (1994-present) 27, 035112 (2015); doi: 10.1063/1.4915074

View online: http://dx.doi.org/10.1063/1.4915074

View Table of Contents: http://scitation.aip.org/content/aip/journal/pof2/27/3?ver=pdfcov

Published by the AIP Publishing

\section{Articles you may be interested in}

Vertical dispersion of light inertial particles in stably stratified turbulence: The influence of the Basset force

Phys. Fluids 22, 013301 (2010); 10.1063/1.3291678

Three-dimensional structure and decay properties of vortices in shallow fluid layers

Phys. Fluids 13, 1932 (2001); 10.1063/1.1374936

Numerical investigation of the scalar probability density function distribution in neutral and stably stratified mixing layers

Phys. Fluids 13, 927 (2001); 10.1063/1.1352622

Numerical investigation of the entrainment and mixing processes in neutral and stably-stratified mixing layers

Phys. Fluids 11, 162 (1999); 10.1063/1.869910

Are flows electromagnetically forced in thin stratified layers two dimensional?

Phys. Fluids 9, 3102 (1997); 10.1063/1.869419

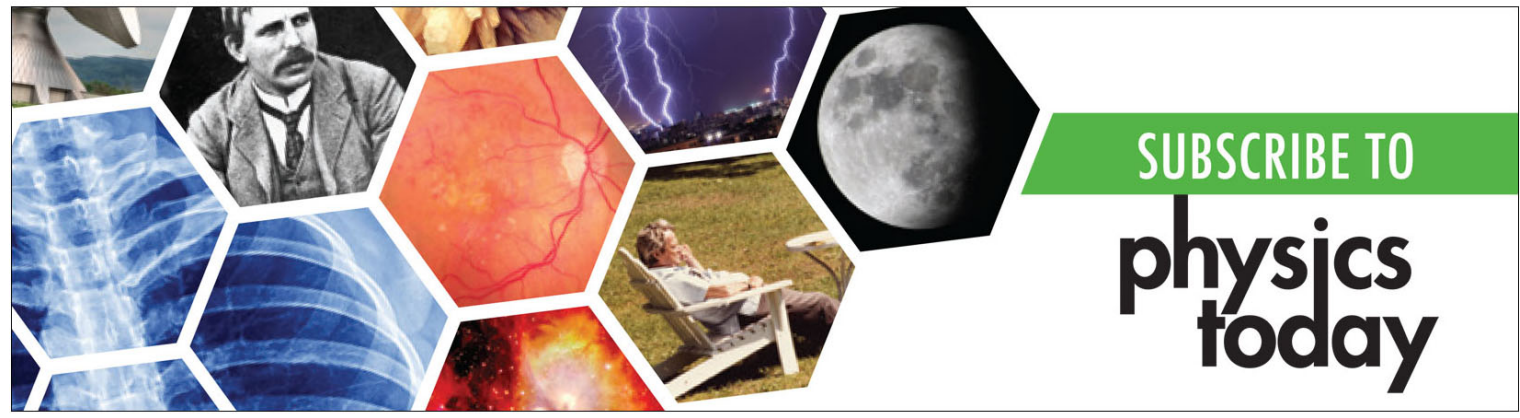




\title{
Dimensional transition of energy cascades in stably stratified forced thin fluid layers
}

\author{
A. Sozza, ${ }^{1}$ G. Boffetta,,${ }^{1, a)}$ P. Muratore-Ginanneschi, ${ }^{2}$ and S. Musacchio ${ }^{3}$ \\ ${ }^{1}$ Department of Physics and INFN, University of Torino, via P. Giuria 1, 10125 Torino, Italy \\ ${ }^{2}$ Department of Mathematics and Statistics, University of Helsinki PL 68, FIN-00014 \\ Helsinki, Finland \\ ${ }^{3}$ Université de Nice Sophia Antipolis, CNRS, LJAD, UMR 7351, 06100 Nice, France
}

(Received 10 September 2014; accepted 4 March 2015; published online 20 March 2015)

\begin{abstract}
We study the effects of a stable density stratification on the turbulent dynamics of thin fluid layers forced at intermediate scales. By means of a set of high-resolution numerical simulations, performed within the Boussinesq approximation, we investigate how the stratification and confinement affect the mechanisms of kinetic and potential energy transfer. The detailed analysis of the statistics of the energy-dissipation rates and energy-exchange rates and of the spectral fluxes of potential and kinetic energy shows that stratification provides a new channel for the energy transfer towards small scales which reduces the large-scale flux of kinetic energy. We also discuss the role of vortex stretching and enstrophy flux in the transfer of kinetic energy into potential energy at small scales. @ 2015 AIP Publishing LLC. [http://dx.doi.org/10.1063/1.4915074]
\end{abstract}

\section{INTRODUCTION}

In many instances of geophysical flows, the fluid motion is confined by material boundaries or other physical mechanisms in thin layers with a small aspect ratio. The thickness of such layers can be much smaller than the typical horizontal scales of motion, while being at the same time much larger than the dissipative viscous scales.

The turbulent dynamics of flows confined in quasi two-dimensional (2D) geometries exhibits a rich and interesting phenomenology. Numerical simulations ${ }^{1,2}$ and experiments ${ }^{3,4}$ have shown that a mixture of 2D and three-dimensional (3D) dynamics can emerge in such situation. In particular, when the thickness of the fluid layer is smaller than the length scale of the forcing, the turbulent cascade of kinetic energy injected by external forces splits in two parts. A fraction of the energy is transferred toward small, viscous scales as in 3D turbulence, while the remnant energy undergoes an inverse cascade toward large scales as in 2D turbulence. The key parameter which determines the energy flux of the two cascades is the ratio $S=L_{z} / L_{f}$ between the confining scale $L_{z}$ and the length scale of the forcing $L_{f} \cdot{ }^{1,2}$

Beside the confinement, other physical factors can affect the effective dimensionality of geophysical flows. One important ingredient is rotation, which typically favors a two-dimensionalization of the flow. ${ }^{5-8}$ The presence of a stable stratification of density also affects the dimensionality of geophysical flows. ${ }^{9}$ In stably stratified rotating flows, an inverse cascade has been observed. ${ }^{10-14}$ In particular, when rotation and stratification have comparable strength (i.e., rotation rate and Brunt-Vaisala frequency are similar), the energy transfer toward large scales is found to be larger than in the purely rotating case. ${ }^{14}$ A typical feature of strongly stratified turbulent flows, which has been observed both in numerical simulations and experiments, ${ }^{11,15-20}$ is the formation of quasi-horizontal layered structure, often called "pancakes." It has also been argued that their formation could be connected to instabilities of columnar vortices. ${ }^{15}$

The presence of these structures is accompanied by a breaking of the isotropy of the flow. In particular, the vertical velocities are strongly suppressed and the horizontal flows become predominant.

a) Author to whom correspondence should be addressed. Electronic mail: boffetta@ to.infn.it 
At the same time, the strong shear between the layered structures originates intense vertical gradients of horizontal velocities. Scaling analysis ${ }^{16,21}$ predicts that the typical thickness of the layered structures $L_{v}$ is proportional to the magnitude of horizontal velocity $U$ and to the inverse of the Brunt-Väisäla frequency $N$. The aspect ratio between the vertical scale $L_{v}$ and horizontal scale $L_{h}$ of the flow is, therefore, proportional to the horizontal Froude number $L_{v} / L_{h} \sim \mathrm{Fr}=U /\left(N L_{h}\right)$.

The role played by these structures on the transfer of energy in stratified turbulence has been extensively investigated because of its relevance for the dynamics of the atmosphere. Early studies proposed the hypothesis that two-dimensional turbulence could develop within the horizontal layers, leading to the formation of an inverse energy cascade which could transfer kinetic energy toward largescale structures. ${ }^{22}$ On the other hand, predictions based on the eddy damped quasi-normal Markovian (EDQNM) closure have argued that the formation of layered structures should block the development of an inverse energy cascade because of the strong dissipation due to the turbulent shear between layers. $^{23}$

Although early simulations of stratified flows reported the presence of a weak inverse cascade, ${ }^{24}$ most numerical simulations have found a direct cascade of kinetic energy. ${ }^{12,14,17,20,21}$ An energy transfer from small to large scale has been observed in numerical simulations for Froude number smaller than a $O(1)$ critical value, ${ }^{11,12,17,21,25}$ but this phenomenon is not associated to an inverse energy cascade of vortical energy as in 2D turbulence. Conversely, kinetic energy piles up in vertically sheared horizontal flows (VSHF), i.e., shear modes with $k_{h}=0, k_{z} \neq 0$. It has been argued that this process is the result of inertial-gravity waves interactions, but its mechanisms are still poorly understood.

The picture which emerges from the results of previous studies is that in the limit of strong stratification, the turbulent flow is characterized by a cascade toward small scales of both kinetic energy and potential energy. ${ }^{20,21}$ These observations pose an intriguing question. Given the fact that in the absence of stratification, the turbulent dynamics of thin fluid layers shows the development of an inverse cascade, which on the contrary is absent in presence of a strong stratification, it is natural to investigate the physical mechanism which bridges these two extreme cases.

In this work, we consider the consequences of stable stratification on a thin layer of turbulent flow and the effect on the direction of the energy cascade. By means of a set of high-resolution numerical simulations, we investigate the mechanisms of transfer of kinetic and potential energies in a stably stratified turbulent flow forced at intermediate scales and confined in fluid layers with variable aspect ratio. We show that the turbulent cascade of potential energy acquired by the fluid acts as a new channel for dissipation that reduces the large-scale flux of kinetic energy and eventually prevents the development of the inverse cascade.

The remaining of the paper is organized as follows: Sec. II presents the governing equation and described the numerical simulations. In Sec. III, we report and discuss the results obtained. Section IV is devoted to conclusions.

\section{GOVERNING EQUATIONS AND NUMERICAL SIMULATIONS}

The equations of motion for an incompressible flow, stably stratified in the vertical direction by a mean density gradient $\gamma$, in the gravitational field $\mathbf{g}=(0,0,-g)$ within the Boussinesq approximation are

$$
\begin{gathered}
\frac{\partial \mathbf{u}}{\partial t}+\mathbf{u} \cdot \boldsymbol{\nabla} \mathbf{u}=-\frac{1}{\rho_{0}} \boldsymbol{\nabla} p+v \nabla^{2} \mathbf{u}-N \phi \mathbf{e}_{3}+\mathbf{f}, \\
\frac{\partial \phi}{\partial t}+\mathbf{u} \cdot \boldsymbol{\nabla} \phi=\kappa \nabla^{2} \phi+N \mathbf{e}_{3} \cdot \mathbf{u},
\end{gathered}
$$

supplemented by the condition of incompressibility $\boldsymbol{\nabla} \cdot \mathbf{u}=0$. Here, $\mathbf{u}=\left(u_{1}, u_{2}, u_{3}\right)$ is the velocity field and $\phi$, which has the dimension of a velocity, is proportional to the deviation of the density field $\rho$ from the linear vertical ( $z$ direction) profile $\rho=\rho_{0}+\gamma(\phi / N-z) \cdot \gamma$ is the mean density gradient which, together with the acceleration of gravity $g$, defines the Brunt-Väisälä frequency $N$ as $N^{2}$ $=\gamma g / \rho_{0}$. The parameters $v$ and $\kappa$ are the molecular viscosity and diffusivity, respectively. The flow 
is sustained by the external force $\mathbf{f}(\mathbf{x}, t)$, which is active on a characteristic correlation scale $L_{f}$ and provides an energy input rate $\varepsilon_{f}$.

In the absence of forcing and dissipation, Eqs. (1) and (2) conserve the total energy density, given by the sum of kinetic and potential contributions

$$
E=E_{k}+E_{p}=\frac{1}{2}\left\langle|\mathbf{u}|^{2}\right\rangle+\frac{1}{2}\left\langle\phi^{2}\right\rangle
$$

where $\langle\ldots\rangle=\frac{1}{V} \int d^{3} x \ldots$ represents the average over the whole volume $V$ of the fluid.

From (1) and (2), the energy balance reads

$$
\frac{d E_{K}}{d t}=\varepsilon_{f}-\varepsilon_{v}-\varepsilon_{x}, \quad \frac{d E_{P}}{d t}=\varepsilon_{x}-\varepsilon_{\kappa},
$$

where we have introduced the viscous dissipation rate of kinetic energy $\varepsilon_{v}=v\left\langle\partial_{i} u_{j} \partial_{i} u_{j}\right\rangle$ (summation over repeated indices is assumed), the diffusive dissipation rate of potential energy $\varepsilon_{\kappa}=\kappa\left\langle\partial_{i} \phi \partial_{i} \phi\right\rangle$, and the exchange rate between kinetic and potential energies $\varepsilon_{x}=N\left\langle u_{3} \phi\right\rangle$. The sign of the exchange rate $\varepsilon_{x}$ is not defined a priori, but the analysis of the Kármán-Howarth-Monin (KHM) equations (discussed in the Appendix) shows that it is positive on average. This indicates that it causes an effective dissipation of kinetic energy and a production of potential energy, which is indeed observed in our simulations.

In the limit of vanishing stratification, $N \rightarrow 0$, the field $\phi$ decouples from the velocity field in (1), which therefore recovers the usual Navier-Stokes equation, while (2) becomes the equation for the evolution of a passive scalar field which decays to zero as it is not forced. In the inviscid limit $(v, \kappa \rightarrow 0)$, both kinetic and potential energies in (3) are invariants.

In the limit $N=0$, the transition from $2 \mathrm{D}$ to $3 \mathrm{D}$ turbulent phenomenology is determined by the aspect ratio $S=L_{z} / L_{f}$ between the vertical scale and the characteristic forcing scale $L_{f} \cdot{ }^{1,2}$ As $S \rightarrow 0$, one recovers the 2D scenario in which the energy flux is towards the large scales $\ell \gg L_{f}$ and kinetic energy grows linearly at a rate $\varepsilon_{\alpha}=d E_{K} / d t$. In the limit of very small viscosity, the standard 2D phenomenology ${ }^{26}$ predicts that $\varepsilon_{\alpha}=\varepsilon_{f}$, i.e., all the injected energy is transferred to large scales. Numerical simulations at increasing values of $S$ have shown a progressive transition toward the 3D phenomenology of direct cascade. ${ }^{2}$ When the vertical scale $L_{z}$ becomes larger than the viscous scale, an intermediate regime with both a direct and an inverse cascade of kinetic energy appears. In this "split cascade" regime, intermediate between 2D and 3D turbulence, a fraction $\varepsilon_{\alpha}<\varepsilon_{f}$ of energy input is transferred toward large scales while the remaining energy is transferred toward small scales where it is dissipated by viscosity at a rate $\varepsilon_{v}$. The conservation of kinetic energy gives a constraint for the sum of the fluxes of the inverse and direct energy cascade $\varepsilon_{\alpha}+\varepsilon_{v}=\varepsilon_{f}$. By increasing the aspect ratio $S$, the ratio $\varepsilon_{v} / \varepsilon_{\alpha}$ increases and eventually a complete 3D phenomenology is recovered in which the system attains a stationary state with $\varepsilon_{\alpha}=0$. The value of $S$ at which the flux of the inverse cascade vanishes is not universal and may depend on the details of the forcing. In the specific numerical setup used in the previous investigations, ${ }^{2}$ this is observed for $S \approx 1 / 2$.

The main goal of our work is to investigate how the above scenario is affected by the presence of a stable stratification of density in the fluid layer. In particular, we will study how the various quantities which appear in the energy balance depend both on the aspect ratio $S$ and the Froude number Fr, and we will determine which part of the parameter space $(S, \mathrm{Fr})$ allows for the development of an inverse energy cascade.

It is worth to notice that the limit of thin aspect ratio $S \rightarrow 0$ and the limit of strong stratification $\mathrm{Fr} \rightarrow 0$ are not expected to commute. In particular, if the limit $\mathrm{Fr} \rightarrow 0$ is taken before $S \rightarrow 0$, it is not obvious that the dynamics of 3D stably stratified turbulence should smoothly become $2 \mathrm{D}$ at vanishing domain height. However, numerical simulations at finite stratification $\mathrm{Fr}>0$, as in our study, are not affected by this issue.

We performed numerical simulations of hyperviscous version of Eqs. (1) and (2) by means of a parallel, fully dealiased, pseudo-spectral code. The flow is confined in a triply periodic domain, with horizontal sizes $L_{x}=L_{y}=2 \pi$ and vertical size $L_{z} \ll L_{x}, L_{y}$. The domain is discretized on a regular grid with resolution $N_{x}=N_{y}=512$ and $N_{z}=N_{x}\left(L_{z} / L_{x}\right)$. 
In the setup of our numerical simulations, the forcing $\mathbf{f}(\mathbf{x}, t)$ which sustains the turbulent flow is a Gaussian, white-in-time stochastic noise, active only on the horizontal components of velocity $\left(u_{1}, u_{2}\right)$, and depending on the horizontal components $(x, y)$ only. This choice is consistent with the two-dimensional limit for $L_{z} \rightarrow 0$ and gives a forcing (and energy input) which is independent of the parameter $L_{z}$. The forcing is localized in the Fourier space in a narrow band of wave numbers $|\mathbf{k}| \simeq k_{f}=8$ and injects energy into the system at a fixed rate $\varepsilon_{f}$. We adopt a hyperviscous damping scheme $v_{p} \nabla^{2 p}$ of order $p=8$ both for the viscosity and the diffusivity, with $v_{p}=\kappa_{p}=1 \times 10^{-33}$.

The non-dimensional parameter which quantify the confinement is given by the ratio

$$
S=\frac{L_{z}}{L_{f}}
$$

between the vertical scale and the characteristic forcing scale $L_{f}=2 \pi / k_{f}$.

The intensity of the stratification is expressed in terms of a second dimensionless number, the Froude number, here and in the following defined as ${ }^{11}$

$$
\mathrm{Fr}=\frac{\varepsilon_{f}^{1 / 3} k_{f}^{2 / 3}}{N} .
$$

It is noteworthy that this definition of the Froude number is designed to non-dimensionalize the parameters which are fixed a priori in the simulations, and it is not based on observables that are measured a posteriori. Under the assumption that the typical horizontal scale of the flow $L_{h}$ is of the order of the forcing scale $L_{f}$, the definition (6) is equivalent to the horizontal Froude number. ${ }^{16}$ The region of parameter space $(S, F r)$ explored in our simulations is shown in Table I.

\section{RESULTS}

For each couple of parameters $(\mathrm{Fr}, S)$, we performed a numerical simulation starting from initial conditions $\mathbf{u}(\mathbf{x}, t=0)=0$ and $\phi(\mathbf{x}, t=0)=0$, that is with the fluid at rest and without density fluctuations. In the first stage ( $t \leq 10 \tau_{f}$ where $\tau_{f} \equiv \varepsilon_{f}^{-1 / 3} k_{f}^{-2 / 3}$ ), the flow is not yet turbulent: small scale energy dissipation is zero and kinetic energy grows at the input rate $\varepsilon_{f}$. After this transient stage, turbulence develops and the potential energy $E_{P}$ attains statistically steady values which increases as Fr is reduced (see Figure 1).

The temporal evolution of the kinetic energy $E_{K}(t)$ is also shown in Figure 1 for different values of Fr. In the turbulent stage, and in the absence of stratification, the energy grows linearly in time, with a rate $d E_{K} / d t=\varepsilon_{\alpha}<\varepsilon_{f}$ that is equal to the flux of the inverse energy cascade. By increasing the stratification, this growth rate reduces, and eventually vanishes (for $\mathrm{Fr} \simeq 0.2$ in this particular case). It is worth noticing that the kinetic energy associated with the vertical motions $\left\langle u_{3}^{2}\right\rangle$ becomes statistically constant for any Fr, therefore the observed growth of kinetic energy is due solely to horizontal flows.

TABLE I. Parameter space of the simulations. Each number corresponds to a simulation and represents the fraction of energy transferred to large scales, $\varepsilon_{\alpha} / \varepsilon_{f}$, as a function of $\mathrm{Fr}=\left(\varepsilon_{f}^{1 / 3} k_{f}^{2 / 3}\right) / N$ and $S=L_{z} / L_{f}$.

\begin{tabular}{llllllllllll}
\hline \hline Fr/S & 0.5 & 0.438 & 0.375 & 0.344 & 0.313 & 0.282 & 0.25 & 0.219 & 0.188 & 0.172 & 0.125 \\
\hline$\infty$ & 0.04 & 0.05 & 0.10 & & 0.19 & & 0.28 & & 0.38 & & 0.54 \\
0.75 & 0.0 & 0.03 & 0.06 & & 0.18 & & 0.27 & & 0.34 & 0.53 \\
0.6 & & 0.03 & 0.05 & 0.13 & 0.15 & & 0.26 & & 0.33 & \\
0.5 & & 0.02 & 0.02 & & 0.14 & & 0.25 & & 0.32 & 0.52 \\
0.4 & & & 0.03 & 0.09 & 0.12 & & 0.23 & & 0.31 & 0.51 \\
0.3 & & & & 0.0 & 0.047 & 0.09 & 0.13 & 0.25 & 0.27 & 0.48 \\
0.25 & & & & & 0.0 & 0.04 & 0.11 & 0.20 & 0.21 & & 0.47 \\
0.2 & & & & & & 0.01 & 0.10 & 0.15 & 0.27 & 0.41 \\
0.15 & & & & & & & & 0.0 & 0.12 & 0.23 \\
\hline \hline
\end{tabular}



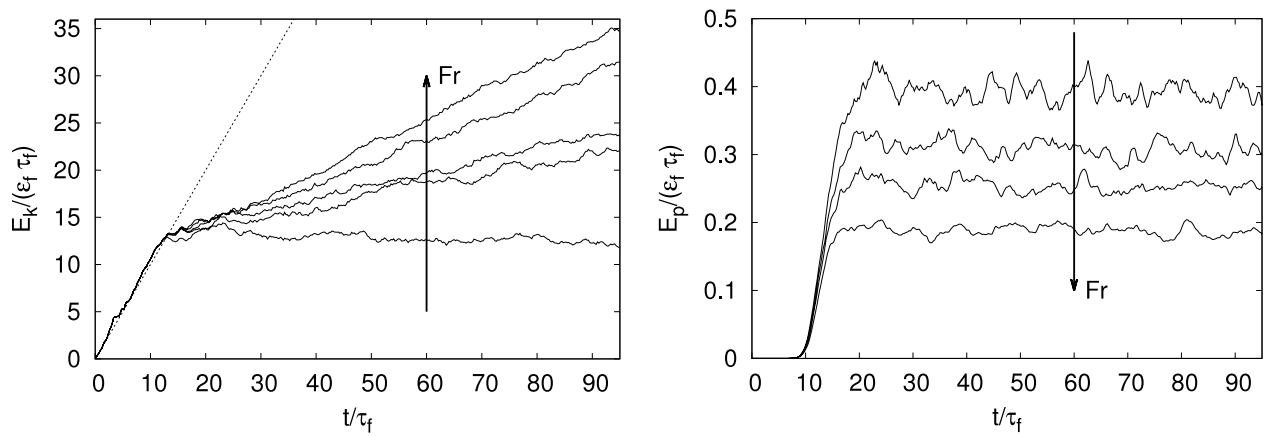

FIG. 1. Temporal evolution of kinetic energy $E_{K}(t)$ (left panel) and potential energy $E_{P}(t)$ (right panel) at $S=0.25$. Froude numbers are $\mathrm{Fr}=0.2,0.25,0.3,0.4$ and $\mathrm{Fr}=\infty$.

In Table I, we report for each simulation the values of the energy growth rates $\varepsilon_{\alpha}$, measured from the growth of $E_{K}(t)$, normalized to the energy input $\varepsilon_{f}$. We recall that this ratio is equal to the fraction of energy that is transferred to large scales, producing the inverse energy cascade.

For fixed values of Fr, the ratio $\varepsilon_{\alpha} / \varepsilon_{f}$ is a decreasing function of the aspect ratio $S$ (see Figure 2), and vanishes for a critical aspect ratio $S_{c}(\mathrm{Fr})$ which becomes smaller as the stratification increases. The behavior of $S_{c}$ (determined by linear interpolation of the lines displayed in Fig. 2) as a function of $\mathrm{Fr}$ is shown in Figure 3. In the limit of vanishing stratification $\mathrm{Fr} \rightarrow \infty$, the critical aspect ratio is bounded by the value $S_{c} \simeq 1 / 2$ which is observed in the absence of stratification. ${ }^{2}$ For sufficiently strong stratification, we find that $S_{c}$ becomes smaller and follows, for small $\mathrm{Fr}$, approximately the scaling $S_{c} \sim$ Fr.

The scaling of the critical aspect ratio $S_{c} \sim$ Fr provides a crucial indication to understand the mechanism which causes the suppression of the inverse energy cascade induced by stratification. In analogy with the aspect ratio $S$, which expresses the ratio between the confining scale $L_{z}$ and the forcing scale $L_{f}$, the Froude number can also be rewritten as the ratio between the characteristic vertical scale of the layered structures that characterize the stratified flows $L_{v}=\varepsilon_{f}^{1 / 3} L_{f}^{1 / 3} / N$ and the forcing scale $L_{f}$

$$
\frac{L_{v}}{L_{f}}=\frac{\varepsilon_{f}^{1 / 3} L_{f}^{-2 / 3}}{N} \simeq \text { Fr. }
$$

The condition to achieve a complete suppression of the inverse cascade $S \simeq$ Fr is, therefore, equivalent to the condition $L_{z} \simeq L_{v}$. This suggests a relation between the formation of layered structures and the

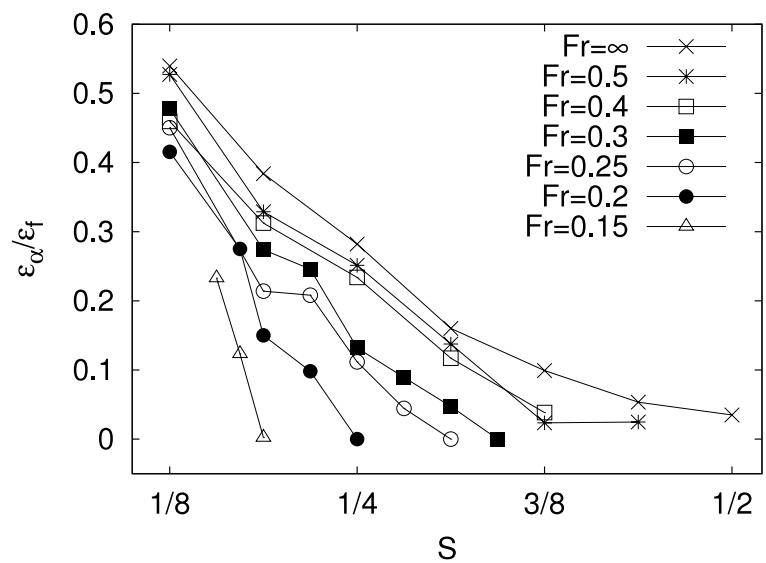

FIG. 2. Growth rates of kinetic energy $\varepsilon_{\alpha}$ (measured from the growth rate of $E_{K}(t)$ ) normalized with the energy input $\varepsilon_{f}$, as a function of the aspect ratio $S=L_{z} / L_{f}$ for different Fr. 


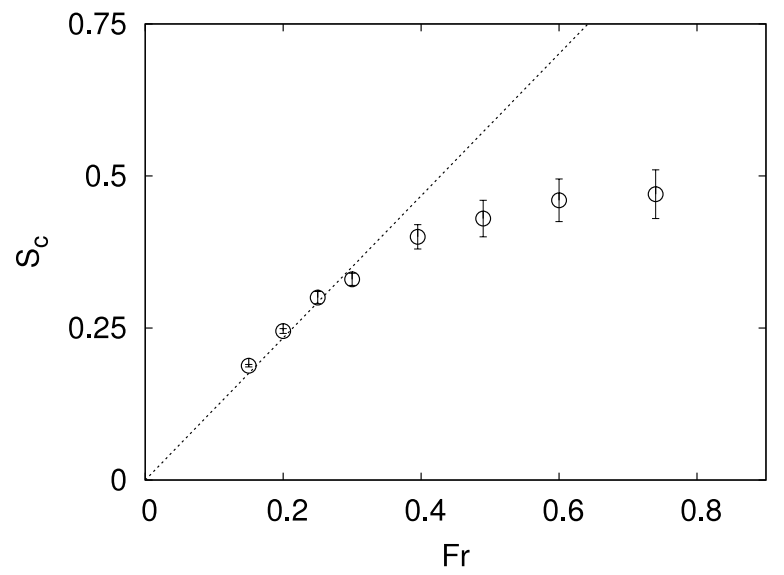

FIG. 3. Critical aspect ratio $S_{c}$, estimated from the data in Fig. 2, as a function of Fr. The line represents the fit $S_{c} \simeq 1.17 \mathrm{Fr}$ obtained over the first four points.

suppression of the inverse cascade. In a layer with a given height $L_{z}$, the inverse cascade disappears when the stratification is sufficiently strong such that the typical thickness of the pancake structures becomes small enough to fit in the fluid layer (i.e., smaller than $L_{z}$ ). From a dynamical point of view, the suppression of the inverse cascade means that the kinetic energy is not transported to large scales and this requires that a different term in the energy transfer becomes relevant.

Let us, therefore, consider the fraction of energy that is transported toward small scales. In Figure 4, we show the dissipation rate of kinetic energy $\varepsilon_{v}$ and potential energy $\varepsilon_{\kappa}$ due, respectively, to the viscosity and molecular diffusivity. Both quantities are normalized with the total dissipation $\varepsilon_{T}$ $=\varepsilon_{v}+\varepsilon_{\kappa}$. In the literature, the quantity $\varepsilon_{\kappa} / \varepsilon_{T}$ is often called mixing efficiency. ${ }^{27}$ In the limit of vanishing stratification $\mathrm{Fr} \rightarrow \infty$, one has trivially $\varepsilon_{v} / \varepsilon_{T} \rightarrow 1$ and $\varepsilon_{K} / \varepsilon_{T} \rightarrow 0$. At increasing stratification, the fraction of energy dissipated by viscosity reduces, while the energy dissipation due to diffusivity grows. Our findings suggest that in the limit of strong stratification $\mathrm{Fr} \rightarrow 0$, the two dissipations may become of the same order $\varepsilon_{v} \simeq \varepsilon_{\kappa}$, and therefore the mixing efficiency $\varepsilon_{\kappa} / \varepsilon_{T}$ could approach asymptotically the value $1 / 2$. It is interesting to note that the ratios $\varepsilon_{v} / \varepsilon_{T}$ and $\varepsilon_{\kappa} / \varepsilon_{T}$ do not show a strong dependence on the aspect ratio $S$.

We remark that, since $\varepsilon_{f}=\varepsilon_{\alpha}+\varepsilon_{T}$, the decrease of $\varepsilon_{\alpha}$ with $\mathrm{Fr}^{-1}$ shown in Fig. 1 corresponds to an increase of total dissipation $\varepsilon_{T}$ with stratification.

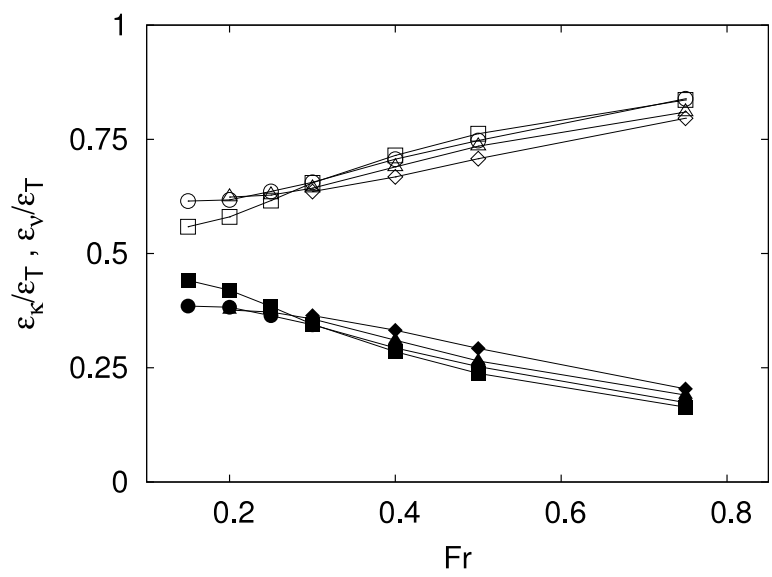

FIG. 4. Kinetic $\varepsilon_{V}$ (open symbols) and potential $\varepsilon_{K}$ (filled symbols) energy dissipation rates normalized by the total dissipation $\varepsilon_{T}=\varepsilon_{v}+\varepsilon_{K}$ as a function of Fr for different $S=0.125$ (squares), $S=0.188$ (circles), $S=0.25$ (triangles), and $S=0.313$ (diamonds). 


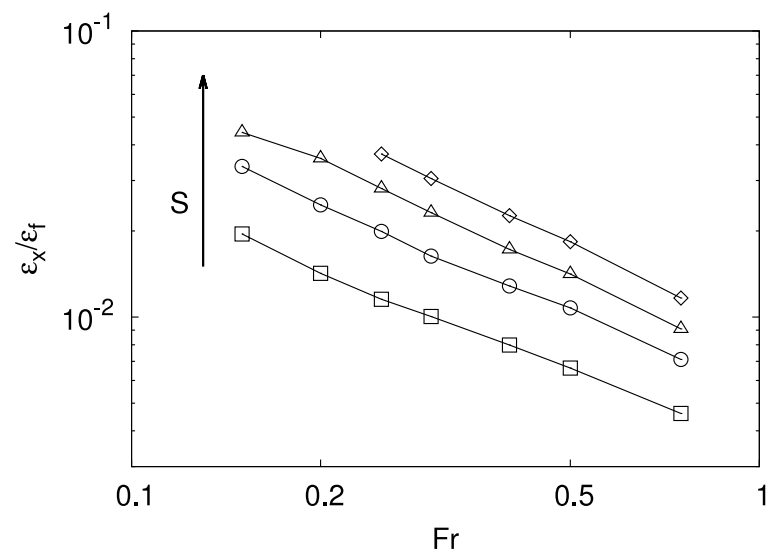

FIG. 5. Exchange rates between kinetic and potential energies $\varepsilon_{x}$ normalized with the energy input $\varepsilon_{f}$, as a function of Fr for different $S=0.125$ (squares), $S=0.188$ (circles), $S=0.25$ (triangles), and $S=0.313$ (diamonds).

The exchange rate from kinetic to potential energy $\varepsilon_{x}=N\left\langle u_{3} \phi\right\rangle$ is shown in Figure 5. The sign of $\varepsilon_{x}$ is always positive, indicating that there is an irreversible conversion of kinetic energy into potential energy that is dissipated by the turbulent diffusivity. We find that the exchange rate decays approximately as $\varepsilon_{x} \sim \mathrm{Fr}^{-1}$, and grows as the aspect ratio $S$ is increased.

In Figure 6, we show the probability density function (PDF) of the local exchange rate $\chi \equiv N u_{3} \phi$ (with $\varepsilon_{x}=\langle\chi\rangle$ ) for different values of Fr and $S$. The PDFs are non-Gaussian and they are characterized by broad negative and positive tails. This implies that the mean positive value of $\chi$, which indicates the mean preferential transfer of energy from kinetic to potential, is the result of strong cancellations between local events of intense energy transfer in both directions. These events can be identified with the large-scale vertical oscillations due to internal gravity waves, which originate periodic exchanges between kinetic and potential energies. We find that the asymmetry of the PDFs reduces as Fr decreases, and that the right tail grows as the aspect ratio $S$ is reduced. The intermittent behavior of the energy transfer revealed by the broad tails of the PDFs of the local exchange rate $\chi \equiv N u_{3} \phi$ is in agreement with the recent findings showing that the PDFs of $\phi$ and $u_{3}$ have non-Gaussian behavior. ${ }^{28}$

More detailed information on the mechanism of energy transfer is provided by the inspection of the fluxes of kinetic and potential energies

$$
\begin{aligned}
& \Pi_{K}(k)=\int_{|\boldsymbol{q}| \leq k} d \boldsymbol{q}(\boldsymbol{u} \cdot \nabla \boldsymbol{u})(\boldsymbol{q}) \boldsymbol{u}^{*}(\boldsymbol{q}), \\
& \Pi_{P}(k)=\int_{|\boldsymbol{q}| \leq k} d \boldsymbol{q}(\boldsymbol{u} \cdot \nabla \boldsymbol{\phi})(\boldsymbol{q}) \boldsymbol{\phi}^{*}(\boldsymbol{q}) .
\end{aligned}
$$
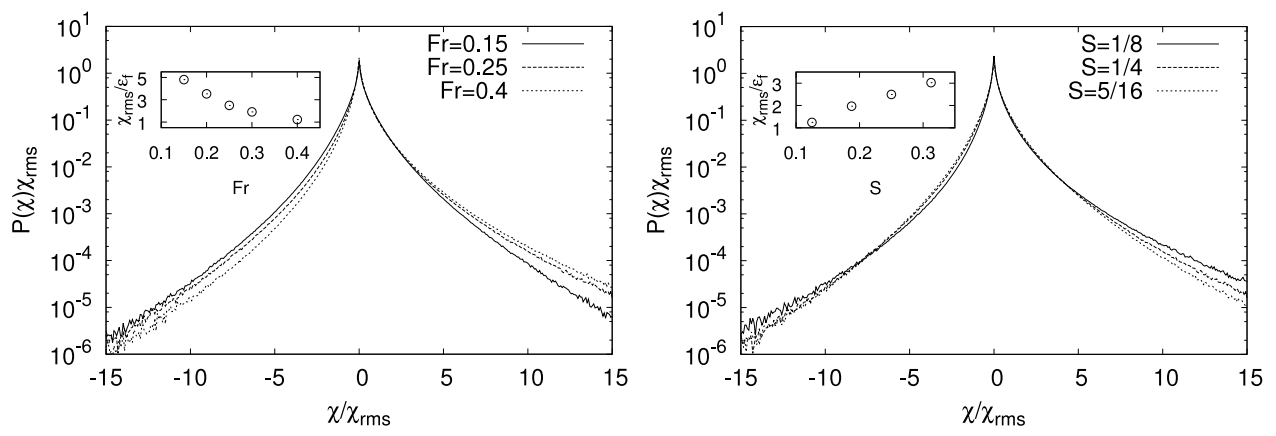

FIG. 6. Probability density functions of the local exchange term $\chi$ (rescaled with the rms value) for different values of Fr at $S=0.25$ (left panel) and different $S$ at $\mathrm{Fr}=0.25$ (right panel). In the insets, the dependence of the rms value $\chi_{\mathrm{rms}}$ on $\mathrm{Fr}$ and $S$ is shown. 

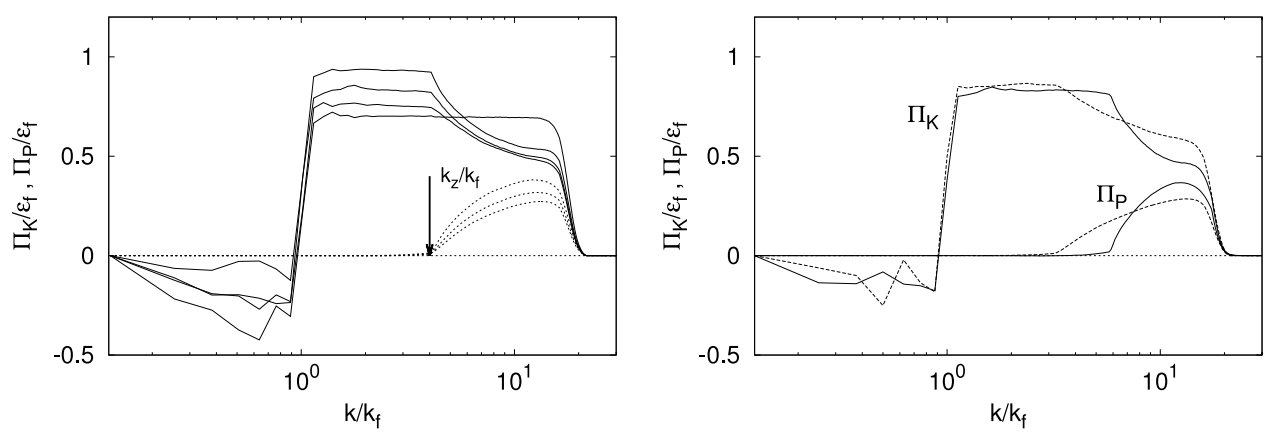

FIG. 7. Spectral fluxes of kinetic energy (solid lines) and potential energy (dotted lines) as a function of the normalized wavenumber $k / k_{f}$. Left panel: Comparison at fixed aspect ratio $S=1 / 4$ and Froude number $\mathrm{Fr}=0.2, \mathrm{Fr}=0.25, \mathrm{Fr}=0.3$, $\mathrm{Fr}=\infty$ from top to bottom. Right panel: $S=0.172, \mathrm{Fr}=0.15$ (solid lines) $S=0.313, \mathrm{Fr}=0.4$ (dotted lines).

In the absence of stratification, the spectral flux of kinetic energy $\Pi_{K}(k)$ displays two plateau (see Figure 7). At small wavenumbers $k<k_{f}$, the energy flux is negative, signaling the presence of an inverse energy cascade. At large wavenumbers $k>k_{f}$, the positive plateau of the flux indicates the direct energy cascade towards small scales.

The presence of a stable stratification of density affects the dual cascade of kinetic energy in both ranges of wavenumbers. At small wavenumbers, the flux of the inverse cascade is reduced with increasing stratification, as Fr decreases. Simultaneously, in the range of wavenumbers $k_{f}<k<k_{z}$, the flux of kinetic energy toward small scales is enhanced.

At large wavenumbers $k>k_{z}$ (larger than the wavenumber associated with the thickness of the fluid layer $k_{z}=2 \pi / L_{z}=k_{f} / S$ ), we observe the development of a cascade of potential energy, whose flux grows as Fr decreases. The direct cascade of potential energy develops at the expenses of the kinetic energy, whose flux shows a significative reduction at large wavenumbers $k>k_{z}$. Remarkably, at very large wavenumbers $k \gg k_{z}$, the fluxes of kinetic energy are always smaller than in the non-stratified case, although they display a weak growth at increasing stratification. This indicates that the main effect of the vertical shears associated with the layered structures in stratified flows is to promote the conversion of kinetic energy into potential energy, rather than to cause an enhancement of the viscous dissipation.

The conversion of kinetic energy into potential energy is a process which requires moving a parcel of fluid in the vertical direction. Its efficiency is, therefore, strongly affected by the thickness of the fluid layer. The space required in the vertical direction to achieve the maximum conversion is of the order of the thickness of the pancake structures $L_{v}$. If the depth of the fluid layer $L_{z}$ is thinner than $L_{v}$, it is possible to achieve only a partial conversion, allowing for the survival of a remnant inverse energy cascade toward large scales. The inverse cascade is completely suppressed when the layer is sufficiently thick or the stratification is sufficiently strong, such that $L_{z}>L_{v}$, allowing for the maximum conversion of kinetic energy into potential energy. This picture is consistent with the scaling $S_{c} \sim$ Fr observed in Figure 3.

It is interesting to note that the same repartition between the large-scale and the small-scale energy transfer can be realized in flows with different parameters. The flux of the inverse energy cascade is determined by two competing mechanisms. The suppression of the enstrophy production due to the vertical confinement favors the inverse cascade. Conversely, the stratification increases the small-scale dissipation of potential energy via the direct cascade of density fluctuations (which is allowed because of the three-dimensionalization of the flow), and therefore reduces the inverse cascade. Because of this competition, a thin and strongly stratified layer can have a similar flux of energy toward large scale than a less confined and less stratified case (see the example in Fig. 7). Nevertheless, the small scale dynamics of these two flows is different, as it is clearly shown by the different behavior at high wavenumbers of the fluxes of kinetic and potential energies.

In the absence of stratification, a crucial role in determining the ratio between the fluxes of kinetic energy of the direct and inverse cascade is played by the phenomenon of vortex stretching. In three-dimensional flows, vortex stretching is responsible for the production of enstrophy, which is 

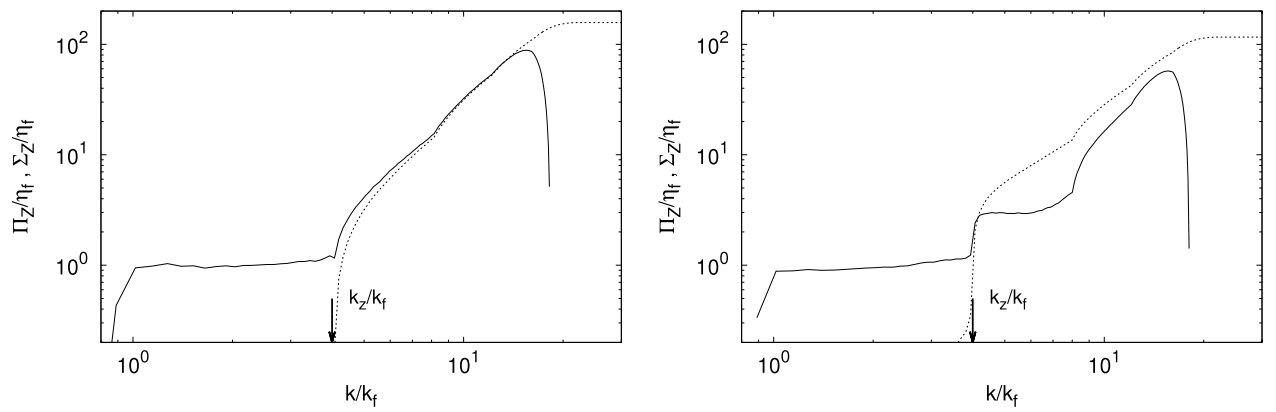

FIG. 8. Spectral flux of enstrophy $\Pi_{Z}$ (solid line) and vortex-stretching $\Sigma_{Z}$ (dashed line) for simulations at $S=0.25$ and $\mathrm{Fr}=\infty$ (left panel) and $\mathrm{Fr}=0.2$ (right panel). All quantities are normalized by the enstrophy input by forcing.

related to the rate of viscous dissipation of energy and therefore to the flux of energy in the direct cascade. Conversely, in ideal two-dimensional flows, the term responsible for the vortex stretching vanishes, and enstrophy becomes an inviscid invariant. The joint conservation of enstrophy and energy causes the reversal of the energy cascade, which is transferred toward large scales.

In the case of a fluid layer with a finite thickness $L_{z}$ smaller than the forcing scale $L_{f}$, we observe the phenomenon shown in Figure 8. The spectral flux of enstrophy $\Pi_{Z}$ and the spectral production of enstrophy $\Sigma_{Z}$ are defined as

$$
\begin{aligned}
& \Pi_{Z}=\int_{|\boldsymbol{q}| \leq k} d \boldsymbol{q}(\boldsymbol{v} \cdot \nabla \omega)(\boldsymbol{q}) \omega^{*}(\boldsymbol{q}), \\
& \Sigma_{Z}=\int_{|\boldsymbol{q}| \leq k} d \boldsymbol{q}(\omega \cdot \nabla \boldsymbol{v})(\boldsymbol{q}) \omega^{*}(\boldsymbol{q}) .
\end{aligned}
$$

In the non-stratified case $(\mathrm{Fr}=\infty)$, the enstrophy flux is constant in the range $k_{f}<k<k_{z}$, indicating the presence of a direct cascade of enstrophy, consistent with a quasi-two-dimensional phenomenology. The enstrophy production is activated only at $k>k_{z}$ where therefore the enstrophy flux is not conserved. In the stratified case ( $\mathrm{Fr}=0.2$ in Figure 8$)$, the enstrophy flux remains unchanged in the range $k_{f}<k<k_{z}=k_{f} / S$. At higher wavenumber, we do not observe a relevant increase of the production of enstrophy with respect to the non-stratified case. On the contrary, the flux of enstrophy is reduced with respect to the non-stratified case, signaling that a fraction of the enstrophy generated by the vortex stretching is spent in converting kinetic energy into potential energy.

Kinetic energy spectra for two different stratification at $S=0.25$ are shown in Fig. 9. Kinetic energy spectra are computed by considering separately the horizontal components and the vertical component of the velocity field and are plotted as a function of the horizontal components of the wavenumber for different values of the parameters. We remark that even for $\mathrm{Fr}=\infty$, the kinetic energy
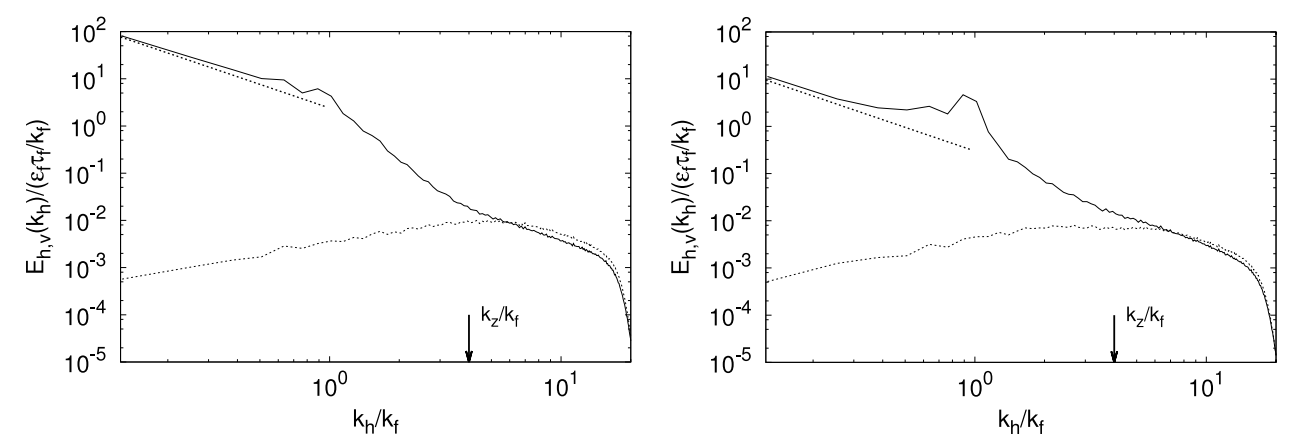

FIG. 9. Horizontal (continuous) and vertical (dotted) kinetic energy spectra as a function of the horizontal wavenumber $k_{h}$ normalized with the forcing wavenumber $k_{f}$ and at $k_{z}=0$ for $S=0.25, \mathrm{Fr}=\infty$ (left panel) and $S=0.25, \mathrm{Fr}=0.15$ (right panel). The dashed lines represent the Kolmogorov scaling $k_{h}^{-5 / 3}$. The arrows indicate the value of the inverse thickness $k_{z}$. 
spectrum at large scales is dominated by the horizontal components, as a consequence of the vertical confinement (and forcing). For scales larger than the forcing, the horizontal spectrum is compatible with Kolmogorov scaling, confirming the presence of an inverse energy cascade suggested by the fluxes in Fig. 7. At scales smaller than the vertical confinement $\left(k>k_{z}\right)$, the spectrum almost recovers isotropy. In the presence of stratification, $\mathrm{Fr}=0.15$, the horizontal spectrum at large scales is reduced, consistently with the reduced flux discussed above. At small scales, both horizontal and vertical spectra are reduced with respect to the $\mathrm{Fr}=\infty$ case because part of kinetic energy is converted into potential energy.

\section{CONCLUSIONS}

We have investigated the direction of the energy flux in a set of numerical simulations of a thin layer of stratified fluid for different values of stratification (Froude number Fr) and thickness (aspect ratio $S$ ). We have shown that, in general, stratification reduces the intensity of the inverse cascade and, consequently, the critical aspect ratio $S_{c}$ at which the inverse flux vanishes.

For small values of Fr, this critical number is found to grow approximately as $S_{c} \simeq$ Fr. This fact supports the picture by which the inverse cascade vanishes when the thickness of the pancake structures in the flow is sufficiently small to fit into the fluid layer. For larger values of Fr, the critical aspect ratio is found to recover the unstratified limit.

A spectral analysis of the kinetic and potential energy fluxes shows that the suppression of the inverse cascade of kinetic energy is accompanied by the generation of a direct cascade of potential energy which becomes an alternative channel for the transfer and dissipation of injected energy.

Our findings open new perspectives for experimental and theoretical research in turbulence. Stable stratification of density has been often used in experimental setup of electromagnetically forced thin fluid layers with the purpose of suppressing vertical motions and enhancing the twodimensionality of the flow. It would be interesting to observe experimentally the suppression of the inverse cascade at increasing stratifications, and to compare the results with our numerical findings. We remark that in our numerical simulations, the confinement in the vertical direction is imposed by the periodic boundary conditions. In a stably stratified flow, such conditions impose the reconnection of the layered structures in the vertical direction. We expect that different boundary conditions, relevant for experimental flows and applications, would give results, such as the critical aspect ratio, which quantitatively differ from our findings in periodic domains. Nonetheless, we expect that, if the effects of the boundaries are limited to a narrow layer of the domain, our main findings will survive at least at a qualitative level.

From a theoretical point of view, it would be extremely useful to have a predictive phenomenological model which allows one to determine the fraction of energy drained by the direct cascade of potential energy, and therefore to have a quantitative prediction for the suppression of the inverse energy cascade as a function of both the aspect ratio and the stratification.

\section{ACKNOWLEDGMENTS}

Authors are grateful to Filippo De Lillo and Enrico Deusebio for fruitful discussions. The numerical simulations were performed on the INFN Turbofarm cluster in Turin, Italy.

\section{APPENDIX: KÁRMÁN-HOWARTH-MONIN EQUATION FOR STRATIFIED TURBULENCE}

In this appendix, we derive a set of generalized KHM equation for stably stratified turbulence.

We define the velocity and scalar two-point correlation functions as

$$
\begin{gathered}
C_{2}^{(u)}(\mathbf{x}, t) \equiv\langle\mathbf{u}(\mathbf{x}, t) \cdot \mathbf{u}(\mathbf{0}, t)\rangle, \\
C_{2}^{(\phi)}(\mathbf{x}, t) \equiv\langle\phi(\mathbf{x}, t) \phi(\mathbf{0}, t)\rangle,
\end{gathered}
$$

and the correlation among the two fields, representing the exchange of energy between kinetic and potential 


$$
C_{1,1}^{\left(u_{3}, \phi\right)}(\mathbf{x}, t) \equiv\left\langle u_{3}(\mathbf{x}, t) \phi(\mathbf{0}, t)\right\rangle .
$$

Dissipation of kinetic and potential energies is defined as $(\alpha, \beta=1,2,3$ and we sum over repeated index)

$$
\begin{gathered}
D^{(u)}(\mathbf{x}, t) \equiv 2 v\left\langle\left(\partial_{\alpha} u_{\beta}\right)(\mathbf{x}, t)\left(\partial_{\alpha} u_{\beta}\right)(\mathbf{0}, t)\right\rangle, \\
D^{(\phi)}(\mathbf{x}, t) \equiv 2 \kappa\left\langle\left(\partial_{\alpha} \phi\right)(\mathbf{x}, t)\left(\partial_{\alpha} \phi\right)(\mathbf{0}, t)\right\rangle,
\end{gathered}
$$

and cross dissipation as

$$
D^{(u, \phi)}(\mathbf{x}, t) \equiv 2 v\left\langle\left(\partial_{\alpha} \phi\right)(\mathbf{x}, t)\left(\partial_{\alpha} u_{3}\right)(\mathbf{0}, t)\right\rangle+2 \kappa\left\langle\left(\partial_{\alpha} u_{3}\right)(\mathbf{x}, t)\left(\partial_{\alpha} \phi\right)(\mathbf{0}, t)\right\rangle .
$$

The correlation of the two-dimensional, two-component forcing $\mathbf{f}(\mathbf{x}, t)$ is given by

$$
\left\langle f_{i}(\mathbf{x}, t) f_{j}(\mathbf{0}, 0)\right\rangle=\delta_{i j} \delta(t) F\left(r_{h} / L_{f}\right),
$$

where $i, j=1,2$ and $r_{h}^{2}=x_{1}^{2}+x_{2}^{2}$ is the separation on the horizontal plane and $F(x)$ is the spatial correlation.

The Kármán-Howarth-Monin equations will involve also third-order structure functions. In particular, we define

$$
\begin{gathered}
\mathbf{S}_{3}^{(u)}(\mathbf{x}, t) \equiv\left\langle[\mathbf{u}(\mathbf{x}, t)-\mathbf{u}(\mathbf{0}, t)]|\mathbf{u}(\mathbf{x}, t)-\mathbf{u}(\mathbf{0}, t)|^{2}\right\rangle, \\
\mathbf{S}_{1,2}^{(u, \phi)}(\mathbf{x}, t) \equiv\left\langle[\mathbf{u}(\mathbf{x}, t)-\mathbf{u}(\mathbf{0}, t)][\phi(\mathbf{x}, t)-\phi(\mathbf{0}, t)]^{2}\right\rangle, \\
\mathbf{S}_{2,1}^{\left(u_{3}, \phi\right)}(\mathbf{x}, t) \equiv\left\langle[\mathbf{u}(\mathbf{x}, t)-\mathbf{u}(\mathbf{0}, t)]\left[u_{3}(\mathbf{x}, t)+\phi(\mathbf{x}, t)-u_{3}(\mathbf{0}, t)-\phi(\mathbf{0}, t)\right]^{2}\right\rangle .
\end{gathered}
$$

Starting from (1) and (2), by exploiting homogeneity and incompressibility, we derive a set of generalized KHM equations, the first for kinetic energy

$$
\frac{\partial C_{2}^{(u)}(\mathbf{x}, t)}{\partial t}+D^{(u)}(\mathbf{x}, t)+N C_{1,1}^{\left(u_{3}, \phi\right)}(\mathbf{x}, t)=F(\mathbf{x})+\frac{1}{2} \nabla \cdot \mathbf{S}_{3}^{(u)}(\mathbf{x}, t)
$$

and the second for potential energy

$$
\frac{\partial C_{2}^{(\phi)}(\mathbf{x}, t)}{\partial t}+D^{(\phi)}(\mathbf{x}, t)-N C_{1,1}^{\left(u_{3}, \phi\right)}(\mathbf{x}, t)=\frac{1}{2} \boldsymbol{\nabla} \cdot \mathbf{S}_{1,2}^{(u, \phi)}(\mathbf{x}, t) .
$$

At variance with the usual Navier-Stokes equation, ${ }^{29}$ (A11) involves the additional term $C_{1,1}^{\left(u_{3}, \phi\right)}$ which represents the transfer of energy from kinetic to potential term. The equivalent of the KHM equation for the energy exchange reads

$$
\frac{\partial C_{1,1}^{\left(u_{3}, \phi\right)}(\mathbf{x}, t)}{\partial t}+D^{\left(u_{3}, \phi\right)}(\mathbf{x}, t)-2 N C_{2}^{\left(u_{3}\right)}(\mathbf{x}, t)+2 N C_{2}^{(\phi)}(\mathbf{x}, t)=\frac{1}{2} \boldsymbol{\nabla} \cdot \mathbf{S}_{2,1}^{(u, \phi)}(\mathbf{x}, t) .
$$

Adding up (A11) and (A12), this energy exchange terms cancel and we get the KHM relation for the total energy

$$
\begin{aligned}
& \frac{\partial}{\partial t}\left(C_{2}^{(u)}(\mathbf{x}, t)+C_{2}^{(\phi)}(\mathbf{x}, t)\right)+\left(D^{(u)}(\mathbf{x}, t)+D^{(\phi)}(\mathbf{x}, t)\right)= \\
& F(\mathbf{x})+\frac{1}{2} \boldsymbol{\nabla} \cdot\left(\mathbf{S}_{3}^{(u)}(\mathbf{x}, t)+\mathbf{S}_{1,2}^{(u, \phi)}(\mathbf{x}, t)\right) .
\end{aligned}
$$

For $\mathbf{x} \rightarrow 0$, at finite $v$ and $\kappa$, (A14) gives the energy balance equation

$$
\frac{d}{d t}\left(E_{k}+E_{p}\right)+\varepsilon_{v}+\varepsilon_{\kappa}=\varepsilon_{f}
$$

where $\varepsilon_{f}=F(0) / 2$ is the energy input and $\varepsilon_{v}$ and $\varepsilon_{\kappa}$ are the viscous and diffusive energy dissipations, respectively. In the absence of stratification, the usual Navier-Stokes equations in 3D reach a steady state in which $\varepsilon_{v}=\varepsilon_{f}$ and $d E / d t=0$. This is not the case in 2D, where the inverse cascade transfers kinetic energy to large scales where viscous dissipation is negligible, at least for finite times (of the order of large scale eddy turnover time) as asymptotically in 2D energy is bounded for any finite value 
of viscosity. ${ }^{30}$ Previous investigations and our numerical simulations show that also in the presence of stratification, the total energy reaches a steady state, indicating the absence of an inverse cascade. Therefore, in the following, we will assume stationarity.

Starting from Eq. (A11) and assuming stationarity and dissipative anomaly for the kinetic energy in the limit of vanishing viscosity, one gets

$$
\lim _{v \rightarrow 0} \lim _{|\mathbf{x}| \rightarrow 0}\left\{D^{(u)}(\mathbf{x})+N C_{1,1}^{\left(u_{3}, \phi\right)}(\mathbf{x})-F(\mathbf{x})\right\}=0
$$

which means that the energy input is partly dissipated and partly transferred to the potential energy

$$
D^{(u)}(\mathbf{0})+N C_{1,1}^{\left(u_{3}, \phi\right)}(\mathbf{0})=F(\mathbf{0}) .
$$

Furthermore, taking the same limits of Eq. (A11) but now in inverse order yields

$$
\frac{1}{2} \lim _{|\mathbf{x}| \rightarrow 0} \lim _{v \rightarrow 0} \boldsymbol{\nabla} \cdot \mathbf{S}_{3}^{(u)}(\mathbf{x}, t)=-\left\{F(\mathbf{0})-N C_{1,1}^{\left(u_{3}, \phi\right)}(\mathbf{0})\right\} \leq 0 .
$$

The negative value of the divergence is the hallmark of the direct energy cascade. The relation is thus in agreement with the presence exhibited in Figure 7 of a direct energy cascade for any value of Fr.

In a similar way, assuming stationarity and dissipative anomaly for the potential energy in the limit of vanishing diffusivity in Eq. (A12), one gets

$$
\lim _{\kappa \rightarrow 0} \lim _{|\mathbf{x}| \rightarrow 0}\left\{D^{(\phi)}(\mathbf{x})-N C_{1,1}^{\left(u_{3}, \phi\right)}(\mathbf{x})\right\}=0
$$

and hence

$$
D^{(\phi)}(\mathbf{0})=N C_{1,1}^{\left(u_{3}, \phi\right)}(\mathbf{0}) \geq 0 .
$$

This means that the cross-correlation $C_{1,1}^{\left(u_{3}, \phi\right)}$, being equal to the dissipation of potential energy, is positive definite. Therefore, it acts as a source term for the potential energy and as a dissipation for the kinetic energy.

${ }^{1}$ L. Smith, J. Chasnov, and F. Waleffe, “Crossover from two-to three-dimensional turbulence,” Phys. Rev. Lett. 77, 2467 (1996).

2 A. Celani, S. Musacchio, and D. Vincenzi, “Turbulence in more than two and less than three dimensions," Phys. Rev. Lett. 104, 184506 (2010).

${ }^{3}$ M. Shats, D. Byrne, and H. Xia, “Turbulence decay rate as a measure of flow dimensionality," Phys. Rev. Lett. 105, 264501 (2010).

${ }^{4}$ H. Xia, D. Byrne, G. Falkovich, and M. Shats, “Upscale energy transfer in thick turbulent fluid layers," Nat. Phys. 7, 321-324 (2011).

${ }^{5}$ L. Smith and F. Waleffe, "Transfer of energy to two-dimensional large scales in forced, rotating three-dimensional turbulence," Phys. Fluids 11, 1608-1622 (1999).

${ }^{6}$ E. Lindborg, "The effect of rotation on the mesoscale energy cascade in the free atmosphere," Geophys. Res. Lett. 32, L01809, doi:10.1029/2004GL021319 (2005).

${ }^{7}$ A. Pouquet, A. Sen, D. Rosenberg, P. Mininni, and J. Baerenzung, "Inverse cascades in turbulence and the case of rotating flows," Phys. Scr. T155, 014032 (2013).

${ }^{8}$ E. Deusebio, G. Boffetta, E. Lindborg, and S. Musacchio, "Dimensional transition in rotating turbulence," Phys. Rev. E 90, 023005 (2014).

${ }^{9}$ J. Riley and M. Lelong, "Fluid motions in the presence of strong stable stratification," Annu. Rev. Fluid Mech. 32, 613-657 (2000).

${ }^{10}$ O. Métais, P. Bartello, E. Garnier, J. Riley, and M. Lesieur, “Inverse cascade in stably stratified rotating turbulence,” Dyn. Atmos. Oceans 23, 193-203 (1996).

${ }^{11}$ L. Smith and F. Waleffe, "Generation of slow large scales in forced rotating stratified turbulence," J. Fluid Mech. 451, 145-168 (2002).

${ }^{12}$ M. Waite and P. Bartello, "Stratified turbulence generated by internal gravity waves," J. Fluid Mech. 546, 313-339 (2006)

${ }^{13}$ M. Waite and P. Bartello, "The transition from geostrophic to stratified turbulence," J. Fluid Mech. 568, 89-108 (2006).

${ }^{14}$ R. Marino, P. Mininni, D. Rosenberg, and A. Pouquet, "Inverse cascades in rotating stratified turbulence: Fast growth of large scales," Europhys. Lett. 102, 44006 (2013).

${ }^{15}$ P. Billant and J.-M. Chomaz, "Experimental evidence for a new instability of a vertical columnar vortex pair in a strongly stratified fluid," J. Fluid Mech. 418, 167-188 (2000).

${ }^{16}$ P. Billant and J.-M. Chomaz, "Self-similarity of strongly stratified inviscid flows," Phys. Fluids 13, 1645-1651 (2001).

${ }^{17}$ M. Waite and P. Bartello, "Stratified turbulence dominated by vortical motion," J. Fluid Mech. 517, 281-308 (2004).

${ }^{18}$ R. Godoy-Diana, J. Chomaz, and P. Billant, "Vertical length scale selection for pancake vortices in strongly stratified viscous fluids," J. Fluid Mech. 504, 229-238 (2004). 
${ }^{19}$ O. Praud, A. Fincham, and J. Sommeria, “Decaying grid turbulence in a strongly stratified fluid,” J. Fluid Mech. 522, 1-33 (2005).

${ }^{20}$ G. Brethouwer, P. Billant, E. Lindborg, and J.-M. Chomaz, "Scaling analysis and simulation of strongly stratified turbulent flows," J. Fluid Mech. 585, 343-368 (2007).

${ }^{21}$ E. Lindborg, "The energy cascade in a strongly stratified fluid," J. Fluid Mech. 550, 207-242 (2006).

${ }^{22}$ D. Lilly, "Stratified turbulence and the mesoscale variability of the atmosphere," J. Atmos. Sci. 40, 749-761 (1983).

${ }^{23}$ F. Godeferd and C. Cambon, "Detailed investigation of energy transfers in homogeneous stratified turbulence," Phys. Fluids 6, 2084-2100 (1994).

${ }^{24}$ J. Herring and O. Métais, "Numerical experiments in forced stably stratified turbulence," J. Fluid Mech. 202, 97-115 (1989).

25 J. Laval, J. McWilliams, and B. Dubrulle, "Forced stratified turbulence: Successive transitions with Reynolds number," Phys. Rev. E 68, 036308 (2003).

${ }^{26}$ G. Boffetta and R. Ecke, "Two-dimensional turbulence," Annu. Rev. Fluid Mech. 44, 427-451 (2012).

${ }^{27}$ W. Peltier and C. Caulfield, "Mixing efficiency in stratified shear flows," Ann. Rev. Fluid Mech. 35, 135-167 (2003).

${ }^{28}$ C. Rorai, P. Mininni, and A. Pouquet, "Turbulence comes in bursts in stably stratified flows," Phys. Rev. E 89, 043002 (2014).

${ }^{29}$ U. Frisch, Turbulence: The Legacy of AN Kolmogorov (Cambridge University Press, Cambridge, 1995).

${ }^{30}$ G. L. Eyink, "Exact results on stationary turbulence in 2d: Consequences of vorticity conservation," Physica D 91, 97-142 (1996). 